Mathematical Modelling and Analysis

Volume 22 Number 3, May 2017, 352-372

https://doi.org/10.3846/13926292.2017.1312580

(c) Vilnius Gediminas Technical University, 2017
Publisher: Taylor\&Francis and VGTU

http://www.tandfonline.com/TMMA

ISSN: 1392-6292

eISSN: $1648-3510$

\title{
Reconstruction Algorithms of an Inverse Coefficient Identification Problem for the Schrödinger Equation*
}

\section{Ji-Chuan Liu}

China University of Mining and Technology, School of Mathematics

Xuzhou, Jiangsu 221116, China

E-mail: liujichuan2003@126.com

Received October 9, 2016; revised March 22, 2017; published online May 15, 2017

\begin{abstract}
In this paper, we consider an inverse problem of coefficient identification for the Schrödinger equation from the observation data on the exterior boundary. Our aim is to detect the number, the location, the size and the shape of the coefficient with piecewise constant within a body. This problem is nonlinear and ill-posed, thus we should apply stable and elegant reconstruction algorithms in order to improve the corresponding approximation. We give several examples to show the viability of our proposed methods.
\end{abstract}

Keywords: Schrödinger equation, coefficient identification, Levenberg-Marquardt algorithm, Trust-Region-Reflective optimization algorithm, ill-posed problem.

AMS Subject Classification: 65N20; 65N21.

\section{Introduction}

We consider an inverse problem of coefficient identification for the Schrödinger equation which arises from the problem of detecting the contact resistance of the electronic device [11], such as metal oxide semiconductor field-effect transistors. Inverse coefficient identification problems for the Schrödinger equation have been researched extensively $[14,16,17,18,28,30]$. Uniqueness of this inverse problem was resolved. Kohn and Vogelius $[19,20]$ proved the uniqueness when the coefficient $q$ is piecewise real analytic. Sylvester and Uhlmann [31] proved global uniqueness of the problem when the coefficient $q$ is smooth. Nachman [26] obtained a formula to recover the coefficient $q$ and proved the uniqueness for bounded $q$. Kang [5, 18] obtained the uniqueness result for rough potential $q$. In terms of references $[11,14,18,27,30]$, we know that this inverse coefficient problem is similar to the recovery problem of the conductivity from measurements $[3,6,10,14,22,31]$. In a practical situation, it is impossible to

* The research of Ji-Chuan Liu was supported by the Fundamental Research Funds for the Central Universities (2014QNA57) and the NSF of China (11601512). 
directly measure the contact resistivity to a satisfactory accuracy due to the device miniaturization. So we want to detect the contact resistivity by certain accessible boundary measurements.

To our knowledge, there are many research papers to solve the uniqueness of the inverse coefficient identification problem for the Schrödinger equation. However, there are few papers to care much about how to detect the salient features of the coefficient within a body for the Schrödinger equation. In this paper, we are interested in the numerical algorithms to detect the number of the coefficient with piecewise constant, to determine the location and the size of the coefficient, and to recover the shape of the coefficient for the Schrödinger equation. This inverse problem is nonlinear and ill-posed. That is, the solution, if exists, does not depend continuously on the given Cauchy data. Any small perturbation in the given Cauchy data may cause dramatically large errors to the location, the size and the shape of the coefficient with piecewise constant. To overcome the ill-posedness of an inverse coefficient identification problem, some regularization methods should be employed. Gebauer and Hyvönen [12] applied factorization method to recover the conductivity inside a physical body from boundary measurements of current and voltage for electrical impedance tomography. Ammari et al. [2] proposed boundary element methods to recover the interior shape of a domain that consists of regions of constant conductivity. Duraiswami et al. [9] gave a new algorithm for conductivity imaging to extract more information about the conductivity distribution from data that have been enriched by coupling impedance electrical measurements to localized elastic perturbations. Kress et al. [10,22] proposed regularized Newton iterations algorithm to determine the shape of a perfectly conducting inclusion within a conducting medium.

In this paper, we restrict ourselves to the problem where the coefficient to be imaged consists of sub-domains with constant. Our proposed reconstruction algorithms view this inverse problem as an optimization problem. We can apply iterative techniques to solve this optimization problem. Then this inverse coefficient identification problem is transformed into a transmission problem. Based on the fundamental solution of Laplace equation and helmholtz equation, we can obtain the expression of solution for the transmission problem with boundary integral equations. This inverse problem is nonlinear and illposed. Thus we propose Levenberg-Marquardt algorithm (LMA) and TrustRegion-Reflective optimization algorithm (TRA) to solve this inverse coefficient identification problem.

In order to determine the salient features of the coefficient for the Schrödinger equation, we follow a three-step process: Firstly, we detect the number of the coefficient with piecewise constant. To our knowledge, there are few works to detect the number of the coefficient with piecewise constant [4]. In many practical applications, the number of the coefficient with piecewise constant is given as an input parameter. This is an innovation of our paper. Secondly, we determine the location and the size of the coefficient with piecewise constant along with the number as a priori. From our experiments, we know that our proposed iterative algorithms do not need the starting guess located sufficiently close to the exact solution. Thirdly, we reconstruct the shape of the coefficient with 
piecewise constant along with the number, the location and the size given. $\mathrm{Nu}-$ merical experiments show that the proposed algorithms are feasible and stable to determine the salient features of the coefficient from noise data.

The outline of the paper is as follows. In Section 2, we introduce the inverse coefficient identification problem. In Section 3, we present the parameterization of boundary and the discretization of integral equations. We propose reconstruction algorithms to solve the inverse coefficient identification problem in Section 4. Numerical examples are presented in Section 5 to illustrate the efficiency of the proposed algorithms. Finally, we give some concluding remarks in Section 6.

\section{Formulation of the inverse coefficient identification problem}

In this paper, we consider an inverse coefficient identification problem from the Cauchy data on the exterior boundary of the solution domain. Let $\Omega$ be a smooth bounded domain in $R^{2}$. Our objective is to identify the coefficient $q$ entering the Schrödinger equation

$$
-\Delta u+q u=0, \text { in } \Omega
$$

from the Cauchy data

$$
u=f, \quad \frac{\partial u}{\partial \nu}=g, \text { on } \partial \Omega,
$$

where $\Delta$ is the Laplacian and $\nu$ is the outward unit normal to the boundary $\partial \Omega$.

We will consider an important case of the coefficient, i.e., $q=\sigma \chi(D)$, where $\sigma$ is known which is the value of the contact resistance of the electronic device, $D$ is a sub-domain in $\Omega$ and $\chi(D)$ is the characteristic function of $D$. Our goal is to seek effective and stable algorithms to determine the number, the location, the size and the shape of sub-domains with constant coefficient.

In terms of the boundary measurements, the Dirichlet-to-Neumann map $\Lambda_{q}$ corresponding to the coefficient $q$ is defined by

$$
\Lambda_{q}(f):=g, \text { on } \partial \Omega .
$$

Assume that $q \in L^{p}(\Omega)$ for some $p>2$ and zero is not an eigenvalue of the Dirichlet operator $-\Delta+q$ in $\Omega$. The question of uniqueness for identifying the coefficient $q$ has been extensively studied $[16,18,30,31,32]$. For the boundary inverse problem (2.1) and (2.2), we have the following uniqueness theorem, refer to $[16,18]$.

Theorem 1. Let $q_{1}, q_{2} \in L^{p}(\Omega)$ with $p>2$. If $\Lambda_{q_{1}}=\Lambda_{q_{2}}$, then $q_{1}=q_{2}$.

Because of the piecewise constant coefficient $q$, the inverse coefficient identification problem simplifies into an inverse transmission problem. In the following, we begin to introduce the transmission problem in detail. 
We denote the boundary of $\Omega$ by $\Gamma_{0}:=\partial \Omega$. Assume that $\Omega_{1}, \ldots, \Omega_{N}$ are $N$ simply connected sub-domains contained in $\Omega$, the closures of which are pairwise disjoint, set $D=\bigcup_{k=1}^{N} \Omega_{k}, \Omega_{0}=\Omega \backslash D$. We denote by $\nu$ the outward unit normal to each of the curves $\Gamma_{k}$ for $k=1, \ldots, N$. Furthermore, we set $\Gamma:=\bigcup_{k=1}^{N} \Gamma_{k}$. Figure 1 shows an example with $N=2$.

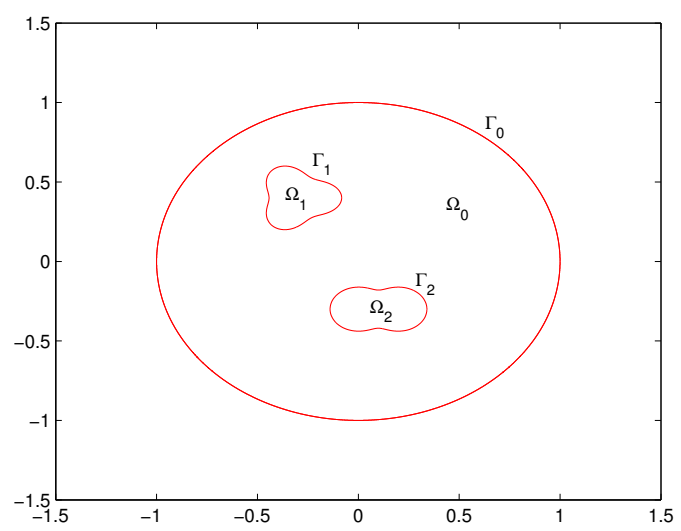

Figure 1. Example of a configuration $\Omega$

According to the choice of the coefficient $q$, the problem (2.1) and (2.2) is equivalent to find piecewise function $u$ satisfying

$$
\begin{aligned}
& -\Delta u^{\text {out }}=0, \text { in } \Omega_{0}, \\
& -\Delta u^{i n}+\sigma u^{i n}=0, \text { in } D
\end{aligned}
$$

and the interface conditions

$$
u^{\text {out }}=u^{\text {in }}, \quad \frac{\partial u^{\text {out }}}{\partial \nu}=\frac{\partial u^{i n}}{\partial \nu}, \text { on } \Gamma_{k}
$$

and the Cauchy data

$$
u^{\text {out }}=f, \quad \frac{\partial u^{\text {out }}}{\partial \nu}=g, \text { on } \Gamma_{0} .
$$

We denote the fundamental solution to the Laplace equation (2.3) by

$$
\Phi^{\text {out }}(x, y)=-\frac{1}{2 \pi} \ln |x-y|, \quad x \neq y
$$

and the Helmholtz equation (2.4) by

$$
\Phi^{i n}(x, y)=\frac{i}{4} H_{0}^{(1)}(i \sqrt{\sigma}|x-y|), \quad x \neq y
$$


where $H_{0}^{(1)}$ is a Hankel function of the first kind of order zero. In terms of the fundamental solution, we have

$$
\begin{aligned}
& u^{\text {out }}(x)=\int_{\Gamma_{0}} \Phi^{\text {out }}(x, y) \varphi_{0}(y) d y+\sum_{k=1}^{N} \int_{\Gamma_{k}} \Phi^{\text {out }}(x, y) \varphi_{k}(y) d y, \quad x \in \Omega_{0}, \\
& u^{\text {in }}(x)=\sum_{k=1}^{N} \int_{\Gamma_{k}} \Phi^{\text {in }}(x, y) \varphi_{N+k}(y) d y, \quad x \in D,
\end{aligned}
$$

where $\varphi_{\widetilde{\ell}}(y)(\widetilde{\ell}=0, \ldots, 2 N)$ are the unknown density on the boundary.

This inverse coefficient identification problem is to determine the number, the location and the size of the coefficient $q$ with piecewise constant, and then try to reconstruct the shape of the coefficient. In practical problems, we can only get the measurement data $g^{\delta}$ which is an approximate function of $g$, and satisfying

$$
\left\|g^{\delta}-g\right\|_{L^{2}\left(\Gamma_{0}\right)} \leq \delta
$$

where $\|\cdot\|_{L^{2}\left(\Gamma_{0}\right)}$ denotes the $L^{2}$-norm on the boundary $\Gamma_{0}$ and the constant $\delta>0$ represents a noisy level.

\section{Parameterization and discretization}

For numerical computations, a parametrization is required. In order to determine the number, the location, the size and the shape of the coefficient with piecewise constant within a body, two methods are introduced to parameterize the boundary $\Gamma_{k}$ of $\Omega_{k}$. Firstly, we parameterize the boundary $\Gamma_{k}$ of $\Omega_{k}$ using polar coordinates as

$$
\Gamma_{k}: O_{k}+\rho_{k}(\cos t, \sin t), 0 \leq t \leq 2 \pi
$$

for determining the number, the location and the size of the coefficient with piecewise constant along with assumptions, where $O_{k}=\left(O_{k, 1}, O_{k, 2}\right)$ is the center of the domain $\Omega_{k}$ and the $\rho_{k}$ is radius.

Let $x_{k}(t)=\left(x_{k, 1}, x_{k, 2}\right)=\rho_{k}(\cos t, \sin t)+O_{k}$, we have

$$
x_{k, 1}^{\prime}=\frac{d x_{k, 1}}{d t}=-\rho_{k} \sin t, \quad x_{k, 2}^{\prime}=\frac{d x_{k, 2}}{d t}=\rho_{k} \cos t .
$$

Then we can obtain the outward unit normal

$$
\nu\left(x_{k}(t)\right)=\frac{\left(x_{k, 2}^{\prime},-x_{k, 1}^{\prime}\right)}{\sqrt{\left(x_{k, 1}^{\prime}\right)^{2}+\left(x_{k, 2}^{\prime}\right)^{2}}}=(\cos t, \sin t) .
$$

We can use $\beta_{k}=\left(O_{k, 1}, O_{k, 2}, \rho_{k}\right)$ to describe the location and the size of the piecewise constant coefficient for an inverse coefficient problem.

Secondly, in order to reconstruct the shape of the coefficient with piecewise constant, we can parameterize the boundary $\Gamma_{k}$ of $\Omega_{k}$ as

$$
\Gamma_{k}: O_{k}+r_{k}(t)(\cos t, \sin t), 0 \leq t \leq 2 \pi
$$


in terms of a $2 \pi$ periodic smooth function $r_{k}(t):[0,2 \pi] \rightarrow(0, \infty)$, where $O_{k}$ is the centroid of the domain $\Omega_{k}$. In our numerical computations, we approximate $r_{k}(t)$ by a trigonometric polynomial of degree less than or equal to $l_{k}$, refer to $[1,22,29]$, i.e.

$$
r_{k}(t)=c_{k, 0}+\sum_{j=1}^{l_{k}}\left[c_{k, j} \cos (j t)+c_{k, j+l_{k}} \sin (j t)\right]
$$

where $c_{k, j} \in R, l_{k} \in N$.

Similarly, let $x_{k}(t)=\left(x_{k, 1}, x_{k, 2}\right)=r_{k}(t)(\cos t, \sin t)+O_{k}$, we know

$$
\begin{gathered}
x_{k, 1}^{\prime}=\frac{d x_{k, 1}}{d t}=r_{k}^{\prime}(t) \cos t-r_{k}(t) \sin t, \\
x_{k, 2}^{\prime}=\frac{d x_{k, 2}}{d t}=r_{k}^{\prime}(t) \sin t+r_{k}(t) \cos t .
\end{gathered}
$$

Thus we can get the outward unit normal vector

$$
\nu\left(x_{k}(t)\right)=\frac{\left(x_{k, 2}^{\prime},-x_{k, 1}^{\prime}\right)}{\sqrt{\left(x_{k, 1}^{\prime}\right)^{2}+\left(x_{k, 2}^{\prime}\right)^{2}}} .
$$

We can use $\beta_{k}=\left(c_{k, 0}, c_{k, 1}, \ldots, c_{k, 2 l}\right)$ to describe the shape of the piecewise constant coefficient for an inverse coefficient problem.

We proceed by describing the discretization of integral equations (2.7) and (2.8). For simplification, we assume the boundary $\Gamma_{0}$ is unit circle that the center is the origin and set $\varphi_{k}(\tau)=\varphi_{k}\left(x_{k}(\tau)\right)$ for $k=0, \ldots, 2 N$. Then we get

$$
\begin{aligned}
& u^{\text {out }}\left(x_{j 1}(t)\right)=\int_{0}^{2 \pi} \Phi^{\text {out }}\left(x_{j 1}(t), x_{0}(\tau)\right) \varphi_{0}(\tau) d \tau \\
& \quad+\sum_{k=1}^{N} \int_{0}^{2 \pi} \Phi^{\text {out }}\left(x_{j 1}(t), x_{k}(\tau)\right) \varphi_{k}(\tau)\left|x_{k}^{\prime}(\tau)\right| d \tau, \quad j 1=0, \ldots, N, \\
& u^{\text {in }}\left(x_{j 2}(t)\right)=\sum_{k=1}^{N} \int_{0}^{2 \pi} \Phi^{i n}\left(x_{j 2}(t), x_{k}(\tau)\right) \varphi_{N+k}(\tau)\left|x_{k}^{\prime}(\tau)\right| d \tau, j 2=1, \ldots, N,
\end{aligned}
$$

where $\varphi_{\tilde{\ell}}(\tau)(\tilde{\ell}=0, \ldots, 2 N)$ are the unknown density on the boundary.

In terms of boundary conditions (2.5) and (2.6), we know that the density $\varphi_{\tilde{\ell}}(\tau)(\tilde{\ell}=0, \ldots, 2 N)$ are the solutions of the following system of integral equations

$$
\begin{aligned}
\int_{0}^{2 \pi} & \Phi^{\text {out }}\left(x_{0}(t), x_{0}(\tau)\right) \varphi_{0}(\tau) d \tau \\
& +\sum_{k=1}^{N} \int_{0}^{2 \pi} \Phi^{\text {out }}\left(x_{0}(t), x_{k}(\tau)\right) \varphi_{k}(\tau)\left|x_{k}^{\prime}(\tau)\right| d \tau=f
\end{aligned}
$$




$$
\begin{aligned}
& \int_{0}^{2 \pi} \Phi^{\text {out }}\left(x_{j 1}(t), x_{0}(\tau)\right) \varphi_{0}(\tau) d \tau+\sum_{k=1}^{N} \int_{0}^{2 \pi} \Phi^{\text {out }}\left(x_{j 1}(t), x_{k}(\tau)\right) \varphi_{k}(\tau)\left|x_{k}^{\prime}(\tau)\right| d \tau \\
& -\sum_{k=1}^{N} \int_{0}^{2 \pi} \Phi^{i n}\left(x_{j 1}(t), x_{k}(\tau)\right) \varphi_{N+k}(\tau)\left|x_{k}^{\prime}(\tau)\right| d \tau=0, \quad j 1=1, \ldots, N . \\
& \int_{0}^{2 \pi} \frac{\partial \Phi^{\text {out }}\left(x_{j 2}(t), x_{0}(\tau)\right)}{\partial \nu\left(x_{j 2}(t)\right)} \varphi_{0}(\tau) d \tau+\sum_{k=1}^{N} \int_{0}^{2 \pi} \frac{\partial \Phi^{\text {out }}\left(x_{j 2}(t), x_{k}(\tau)\right)}{\partial \nu\left(x_{j 2}(t)\right)} \varphi_{k}(\tau) \\
& \quad \times\left|x_{k}^{\prime}(\tau)\right| d \tau+\frac{1}{2} \varphi_{j 2}(t)-\left(\sum_{k=1}^{N} \int_{0}^{2 \pi} \frac{\partial \Phi^{i n}\left(x_{j 2}(t), x_{k}(\tau)\right)}{\partial \nu\left(x_{j 2}(t)\right)} \varphi_{N+k}(\tau)\left|x_{k}^{\prime}(\tau)\right| d \tau\right. \\
& \left.+\frac{1}{2} \varphi_{N+j 2}(t)\right)=0, \quad j 2=1, \ldots, N
\end{aligned}
$$

For the discretization of the integral equations, we note that the second term on the left hand side of (3.2) and the first term on the left hand side of (3.3) are smooth that the trapezoidal rule can be employed for the numerical approximation. However, the first term on the left hand side of (3.2), the second term on the left hand side of (3.3) have a logarithmic singularity, thus we deal with the logarithmic singularity as follows

$$
2 \pi \Phi^{\text {out }}\left(x_{j 1}(t), x_{j 1}(\tau)\right)=-\ln \left|\sin \frac{t-\tau}{2}\right|+\ln \frac{\left|\sin \frac{t-\tau}{2}\right|}{\left|x_{j 1}(t)-x_{j 1}(\tau)\right|}, j 1=0, \ldots, N .
$$

The second term on the right hand side of (3.5) is smooth with diagonal values

$$
\lim _{\tau \rightarrow t} \ln \frac{\left|\sin \frac{t-\tau}{2}\right|}{\left|x_{j 1}(t)-x_{j 1}(\tau)\right|}=-\ln 2\left|x_{j 1}^{\prime}(t)\right|, \quad j 1=0, \ldots, N .
$$

Therefore, the well-estimated quadrature rules for logarithmic singularities are available. We can use the Nyström method to approximate the integral equations with weakly singular kernels in [21]. For (3.4), we know that the second term and the fourth term on the left hand side are smooth with the diagonal values given through the limit

$$
\begin{aligned}
\lim _{\tau \rightarrow t} 2 \pi \frac{\partial \Phi^{\text {out }}\left(x_{j 2}(t), x_{j 2}(\tau)\right)}{\partial \nu\left(x_{j 2}(t)\right)} & =-\lim _{\tau \rightarrow t} \frac{\nu\left(x_{j 2}(t)\right) \cdot\left[x_{j 2}(t)-x_{j 2}(\tau)\right]}{\left|x_{j 2}(t)-x_{j 2}(\tau)\right|^{2}} \\
& =-\frac{\nu\left(x_{j 2}(t)\right) \cdot x_{j 2}^{\prime \prime}(t)}{2\left|x_{j 2}^{\prime}(t)\right|^{2}}, \quad j 2=1, \ldots, N .
\end{aligned}
$$

For the discretization of the integral equations, we should deal the third term and the fourth term on the left hand side of (3.3) and (3.4) using an appropriate method, respectively. Let

$$
M(t, \tau):=\Phi^{i n}\left(x_{j 1}(t), x_{j 1}(\tau)\right)=\frac{i}{4} H_{0}^{(1)}\left(i \sqrt{\sigma}\left|x_{j 1}(t)-x_{j 1}(\tau)\right|\right)
$$

for $t \neq \tau$, we split it into

$$
M(t, \tau)=M_{1}(t, \tau) \ln \left(4 \sin ^{2} \frac{t-\tau}{2}\right)+M_{2}(t, \tau),
$$


where

$$
M_{1}(t, \tau):=-\frac{1}{4 \pi} J_{0}(i \sqrt{\sigma}|z(t)-z(\tau)|)
$$

and the diagonal term for $M_{2}$ is given by

$$
M_{2}(t, t)=\frac{i}{4}-\frac{E}{2 \pi}-\frac{1}{2 \pi} \ln \left(\frac{i \sqrt{\sigma}}{2}\left|z^{\prime}(t)\right|\right),
$$

where $E$ denotes the Euler's constant. Let

$$
\begin{aligned}
L(t, \tau): & =\frac{\partial \Phi^{i n}\left(x_{j 2}(t), x_{j 2}(\tau)\right)}{\partial \nu\left(x_{j 2}(t)\right)} \\
& =\frac{\sqrt{\sigma}}{4} H_{1}^{(1)}\left(i \sqrt{\sigma}\left|x_{j 2}(t)-x_{j 2}(\tau)\right|\right) \frac{\left[x_{j 2}^{\prime}(t)\right]^{\perp} \cdot\left[x_{j 2}(t)-x_{j 2}(\tau)\right]}{\left|x_{j 2}(t)-x_{j 2}(\tau)\right|}
\end{aligned}
$$

which can be decomposed in the form

$$
L(t, \tau)=L_{1}(t, \tau) \ln \left(4 \sin ^{2} \frac{t-\tau}{2}\right)+L_{2}(t, \tau),
$$

where

$$
L_{1}(t, \tau)=\frac{i \sqrt{\sigma}}{4 \pi} J_{1}\left(i \sqrt{\sigma}\left|x_{j 2}(t)-x_{j 2}(\tau)\right|\right) \frac{\left[x_{j 2}^{\prime}(t)\right]^{\perp} \cdot\left[x_{j 2}(t)-x_{j 2}(\tau)\right]}{\left|x_{j 2}(t)-x_{j 2}(\tau)\right|}
$$

and note that the diagonal term $L_{2}(t, t)$ is given by

$$
L_{2}(t, t)=\frac{\left[x_{j 2}^{\prime}(t)\right]^{\perp} \cdot x_{j 2}^{\prime \prime}(t)}{4 \pi\left|x_{j 2}^{\prime}(t)\right|^{2}} .
$$

The interval $[0,2 \pi]$ is partitioned as $0=\tau_{0}<\tau_{1}<\cdots<\tau_{m}=2 \pi$ and $0=t_{0}<t_{1}<\cdots<t_{n}=2 \pi$ where $\tau_{i}=i h_{\tau}(i=0,1, \cdots, m), t_{j}=j h_{t}$ $(j=0,1, \ldots, n)$ and $h_{\tau}=\frac{2 \pi}{m}, h_{t}=\frac{2 \pi}{n}$ are the step sizes. We denote the discrete vector of $\varphi_{k}(\tau)(k=0, \ldots, 2 N)$ as $\Psi_{k}=\left[\varphi_{k}\left(\tau_{0}\right), \varphi_{k}\left(\tau_{1}\right), \ldots, \varphi_{k}\left(\tau_{m-1}\right)\right]^{T}$ and the discrete vectors of $f(t)$ as follows $F=\left[f\left(t_{0}\right), f\left(t_{1}\right), \ldots, f\left(t_{n-1}\right)\right]^{T}$.

Therefore, we can use the well-estimated quadrature rules and the trapezoidal rule to obtain the system of algebraic equations from the system of integral equations (3.2)-(3.4)

$$
A \Psi=b
$$

where

$$
A=\left[\begin{array}{lllllll}
A_{00} & A_{01} & \cdots & A_{0 N} & 0 & \cdots & 0 \\
A_{10} & A_{11} & \cdots & A_{1 N} & A_{1 N+1} & \cdots & A_{12 N} \\
\ddots & & \ddots & & \ddots & & \ddots \\
A_{2 N 0} & A_{2 N 1} & \cdots & A_{2 N N} & A_{2 N N+1} & \cdots & A_{2 N 2 N}
\end{array}\right]
$$

Based on the discretization of the interval $[0,2 \pi]$, the element $A_{j_{1} j_{2}}\left(j_{1}, j_{2}=\right.$ $0, \ldots, 2 N)$ in the matrix $A$ is an $m \times n$ matrix corresponding to an integral in 
the system of integral equations (3.2)-(3.4). We take $m=n$ in our numerical computations for simplicity, thus $\left(A_{j_{1} j_{2}}\right)_{n \times n}$ is a square matrix. For example, $\left(A_{01}\right)_{n \times n}=h_{\tau}\left(\Phi^{\text {out }}\left(x_{0}\left(t_{j}\right), x_{1}\left(\tau_{i}\right)\right) \varphi_{1}\left(\tau_{i}\right)\left|x_{1}^{\prime}\left(\tau_{i}\right)\right|\right)_{n \times n}$ in the second term on the left hand side of (3.2). $\Psi=\left[\Psi_{0}, \Psi_{1}, \ldots, \Psi_{N}, \Psi_{N+1}, \ldots, \Psi_{2 N}\right]^{T}, b=[F, 0, \ldots, 0]^{T}$.

According to (3.1), we can get the flux $g$ on the outer boundary $\Gamma_{0}$

$$
\begin{aligned}
g(t)= & \int_{0}^{2 \pi} \frac{\Phi^{\text {out }}\left(x_{0}(t), x_{0}(\tau)\right)}{\partial \nu\left(x_{0}(t)\right)} \varphi_{0}(\tau) d \tau \\
& +\frac{1}{2} \varphi_{0}(t)+\sum_{k=1}^{N} \int_{0}^{2 \pi} \frac{\partial \Phi^{\text {out }}\left(x_{0}(t), x_{k}(\tau)\right)}{\partial \nu\left(x_{0}(t)\right)} \varphi_{k}(\tau)\left|x_{k}^{\prime}(\tau)\right| d \tau .
\end{aligned}
$$

We denote the discrete vector of $g(t)$ as follows

$$
G=\left[g\left(t_{0}\right), g\left(t_{1}\right), \ldots, g\left(t_{n-1}\right)\right]^{T} .
$$

According to the solution $\Psi$ from (3.6), then we can compute the flux

$$
G=B \Psi,
$$

where $B=\left[B_{00}, B_{01}, \ldots, B_{0 N}, 0, \ldots, 0\right]$. Based on the discretization of the interval $[0,2 \pi]$, the element $B_{0 j_{1}}\left(j_{1}=0, \ldots, N\right)$ in the matrix $B$ is an $m \times n$ matrix corresponding to an integral in integral equation (3.7).

\section{Reconstruction algorithms for the inverse coefficient identification problem}

Our interest is to seek effective reconstruction algorithms to determine the coefficient $q$ with piecewise constant. We know the inverse problem of coefficient identification is severely nonlinear and ill-posed in the sense that quite substantial changes in the coefficient may only result in extremely small changes in the measured data. Therefore, we employ regularization techniques to the proposed reconstruction algorithms for the inverse coefficient identification problem of the Schrödinger equation.

We consider the objective functional

$$
J(\beta)=\frac{1}{2}\left|F(\beta)-g^{\delta}\right|_{L^{2}\left(\Gamma_{0}\right)}^{2}, \quad F(\beta)=\frac{\partial u^{o u t}(\cdot, \beta)}{\partial \nu},
$$

where $g^{\delta}$ are the measured data and

$$
\beta=\left(O_{1,1}, O_{1,2}, \rho_{1}, \ldots, O_{N, 1}, O_{N, 2}, \rho_{N}\right) \in R^{3 N},
$$

or

$$
\beta=\left(c_{1,0}, \ldots, c_{1,2 l_{1}}, \ldots, c_{N, 0}, \ldots, c_{N, 2 l_{N}}\right) \in R^{2\left(l_{1}+\ldots+l_{N}\right)+N} .
$$

The inverse coefficient identification problem is a nonlinear least squares optimization problem. To our knowledge, there are many iterative methods to solve this nonlinear least squares optimization problem. Nonlinear least squares methods involve an iterative improvement to parameter values in order 
to reduce the sum of the squares of the errors between the measured data points and the function. Starting with an initial guess $\beta^{(0)}$, our proposed reconstruction algorithms proceed by the iterations

$$
\beta^{(s+1)}=\beta^{(s)}+\triangle,
$$

where $\triangle$ is the increment vector.

\subsection{Levenberg-Marquardt algorithm (LMA)}

LMA $[23,24]$ is used to solve nonlinear least squares problems which is also known as the damped least-squares method. LMA interpolates between the Gauss-Newton algorithm and the method of gradient descent. LMA is more robust than the Gauss-Newton algorithm, which means that in many cases it finds a solution even if it starts very far off the final minimum. LMA can also be viewed as Gauss-Newton using a trust region approach. LMA is a very popular curve-fitting algorithm used in many software applications for solving generic curve-fitting problems.

The derivative of $F(\beta)$ with respect to the parameters $\beta$ is given by

$$
F^{\prime}(\beta)=\frac{\partial \nabla_{\beta} u^{\text {out }}(\cdot, \beta)}{\partial \nu} .
$$

Therefore, the increment vector $\triangle$ that updates the parameters given by

$$
\triangle=\left(F^{\prime}(\beta)^{T} F^{\prime}(\beta)+\alpha \operatorname{diag}\left(F^{\prime}(\beta)^{T} F^{\prime}(\beta)\right)\right)^{-1} F^{\prime}(\beta)^{T}\left(F(\beta)-g^{\delta}\right),
$$

where $\alpha$ is a regularization parameter. So we can get the final iteration relationship

$$
\beta^{(s+1)}=\beta^{(s)}+\triangle^{(s)} .
$$

For LMA, a key issue is to choose the regularization parameter $\alpha$. A wise choice of regularization parameter is obviously crucial to obtaining useful approximate solutions to ill-posed problems, there are well-studied techniques for computing a good regularization parameter, such as the discrepancy principle [25], the generalized cross-validation (GCV) [13], the L-curve [15] and so on. In this paper, we are interested in a-posteriori rules $\alpha$ for choosing the regularization parameter when minimizing $J(\beta)$. Based on the iteration relationship (4.1), this algorithm for choosing the regularization parameter $\alpha$ is shown in the following.

(1) If $F^{\prime}(\beta)^{T} F^{\prime}(\beta)$ is nonsingular, the iteration process is convergent, then $\alpha=0$.

(2) If $F^{\prime}(\beta)^{T} F^{\prime}(\beta)$ is singular, we take the maximum singular value as $\lambda_{\max }$ and the minimum singular value as $\lambda_{\min }$ by the singular value decomposition. Let the initial regularization parameter $\alpha^{(0)}=\left(\lambda_{\max }+\lambda_{\min }\right) / 2$, to start the iterative process.

(3) In the iterative process, if the value of $J(\beta)$ increases with the number of iterations, iteration stops and returns the modified value of the regularization parameter. In general, we take the half of the the maximum singular value $\lambda_{\max }$ 
and the current regularization parameter as a new regularization parameter, i.e., $\alpha^{(s+1)}=\left(\lambda_{\max }+\alpha^{(s)}\right) / 2$. If the value of $J(\beta)$ decreases with the number of iterations, we take $\alpha^{(s+1)}=\alpha^{(s)}$.

(4) When $J(\beta)$ meets the requirement of computation, for instance, $J(\beta)<$ $\varepsilon$ for a threshold value $\varepsilon$, then the current regularization parameter is optimal.

\subsection{Trust-Region-Reflective optimization algorithm (TRA)}

TRA is a trust region approach for minimizing a nonlinear function subject to simple bounds. TRA does not require that a quadratic programming subproblem, with inequality constraints, be solved in each iteration. Instead, a solution to a trust region subproblem is defined by minimizing a quadratic function subject only to an ellipsoidal constraint which is based on the interior-reflective Newton method described in $[7,8]$. TRA use the method of preconditioned conjugate gradient to solve a large linear system each iteration. The derivative of $F(\beta)$ with respect to the parameters $\beta$ is given as follows

$$
F^{\prime}(\beta)=\frac{\partial \nabla_{\beta} u^{\text {out }}(\cdot, \beta)}{\partial \nu} .
$$

Let the increment $\triangle$ be the solution of the quadratic subproblem

$$
\min _{\triangle \in R^{n}}\left\{\psi(\Delta)=F^{\prime}(\beta)^{T} \triangle+\frac{1}{2} \triangle^{T} M \triangle:|B \triangle| \leq \Lambda\right\},
$$

where $B$ is a positive diagonal scaling matrix, see $[7,8]$, and $\Lambda$ is a positive scalar representing the trust region size, and

$$
M(\beta)=F^{\prime}(\beta)^{T} F^{\prime}(\beta)+B \operatorname{diag}\left(F^{\prime}(\beta)\right) \operatorname{diag}\left(\operatorname{sign}\left(F^{\prime}(\beta)\right)\right) B .
$$

We can get the piecewise linear reflective path $p(\alpha)$ in terms of the initial descent direction $\triangle$. In order to obtain an acceptable step-size $\alpha$, we should minimize the functional $J\left(\beta^{(s)}+p(\alpha)\right)$ with respect to $\alpha$, see [7] for detail. Therefore, we have the final iteration relationship

$$
\beta^{(s+1)}=\beta^{(s)}+p\left(\alpha^{(s)}\right) .
$$

\section{$5 \quad$ Examples}

In this section, we want to examine the applicability of our proposed reconstruction algorithms through some numerical examples. The noisy measured data are generated by

$$
g^{\delta}=g(1+\delta \operatorname{rand}(\operatorname{size}(g)),
$$

where $g$ is the exact data, $\operatorname{rand}(\operatorname{size}(g))$ is a random number uniformly distributed in $[-1,1]$ and the magnitude $\delta$ indicates a relative noise level. In practical applications, the value of the contact resistance of the electronic device is less than $0.1 \mathrm{Ohm}$. In our examples, we take $\sigma=0.01 \mathrm{Ohm}$. 


\subsection{Determining the number of the coefficient with piecewise con- stant}

In order to estimate the number of sub-domains with constant coefficients when $N$ is bounded by a known integer $M$, then we parameterize the boundary $\Gamma_{k}$ of $\Omega_{k}$ as $\Gamma_{k}: O_{k}+\rho_{k}(\cos t, \sin t), 0 \leq t \leq 2 \pi$, along with $\beta_{k}=\left(O_{k, 1}, O_{k, 2}, \rho_{k}\right)$, that is, we use the circle to approximate sub-domains with constant coefficients for every iteration.

We propose two criteria to determine the number of sub-domains with constant coefficients. For a given estimate of the level of noise in $g^{\delta}$, we estimate a threshold value of lower bound $\varepsilon^{\delta}$. Assume a set of circles $\left\{O_{k}\right\}, k \in Z^{+}$, if for every $k_{1} \in Z^{+}$, there exists a $k_{2} \in Z^{+}$such that the Hausdorff distance between the two circles satisfies $d\left(O_{k_{1}}, O_{k_{2}}\right)<\varepsilon^{\delta}$, we call the set $\left\{O_{k}\right\}$ is connected. Our proposed two criteria are the following:

Criterion 1: on the one hand, the circle, whose radius is less than $\varepsilon^{\delta}$, does not take as a sub-domain with piecewise constant coefficient. On the other hand, the circle, whose radius is bigger than $\varepsilon^{\delta}$, is viewed as a sub-domain with piecewise constant coefficient.

Criterion 2: the set of circles, which is connected, can be viewed as a subdomain with piecewise constant coefficient.

Based on the above criteria, we can determine the number $N$ of sub-domians with piecewise constant coefficients according to the threshold value $\varepsilon^{\delta}$.

Example 1. In this case, we suppose the coefficient is a peanut whose polar radius is parameterized by

$$
r(t)=\frac{8}{25} \sqrt{(\cos t)^{2}+\left(\frac{\sin t}{2}\right)^{2}}, 0 \leq t \leq 2 \pi .
$$

Assume the upper bound is $M=2$ as the possible number of the coefficient with piecewise constant. The results are shown in Figure 2

Table 1. The reconstructed radius $\rho$ of different cases with two circles in Figure 2 for Example 1

\begin{tabular}{lll}
\hline$\rho$ & $\rho_{1}$ & $\rho_{2}$ \\
\hline$\rho^{(0)}$ & 0.02 & 0.02 \\
$(a)$ & 0.0244 & 0.2457 \\
$(b)$ & 0.1830 & 0.1445 \\
$(c)$ & 0.0000 & 0.2933 \\
$(d)$ & 0.2511 & 0.0001 \\
\hline
\end{tabular}

TRA is employed to detect the number of the coefficient with piecewise constant along with 0.01 noise level. We take the radiuses $\rho^{(0)}=0.02$ as the starting guess for every sub-domain with constant. We can use the iteration relationship (4.2) to update the $\beta$. From Figure 2 and Table 1, we take the parameters threshold value $\varepsilon^{\delta}=0.025,0.0001,0.0002$, then we can obtain the 


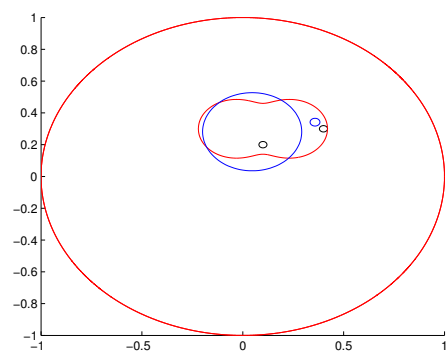

(a) $\rho^{(0)}=0.02$

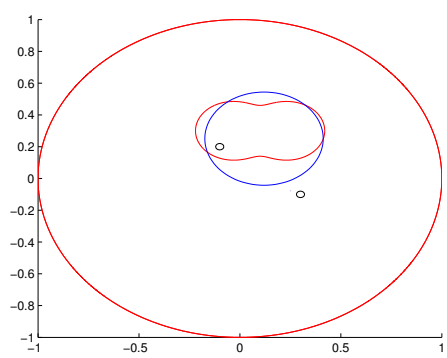

(c) $\rho^{(0)}=0.02$

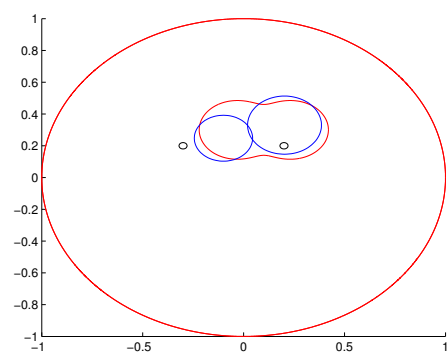

(b) $\rho^{(0)}=0.02$

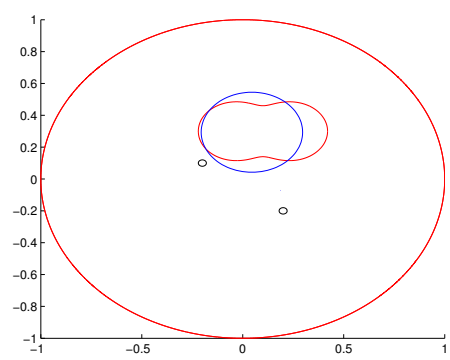

(d) $\rho^{(0)}=0.02$

Figure 2. Apply TRA to detect the number of the coefficient with piecewise constant along with exact solution (red), initial guess (black) and recovered solution (blue), respectively for Example 1

number $N=1$ of sub-domains with the proposed Criterion 1 for Figures 2 (a), (c) and (d), respectively. In Figure 2 (b), two circles intersect which can be seen as a sub-domain in terms of the proposed Criterion 2. We can obtain the same results as TRA by LMA.

\subsection{Determining the location and the size of the coefficient with piecewise constant}

We employ LMA and TRA to determine the location and the size of the coefficient with piecewise constant. In this sub-section, we assume that the number of sub-domains with constant coefficient is given. We parameterize the boundary $\Gamma_{k}$ of $\Omega_{k}$ as $\Gamma_{k}: O_{k}+\rho_{k}(\cos t, \sin t), 0 \leq t \leq 2 \pi$, along with $\beta_{k}=\left(O_{k, 1}, O_{k, 2}, \rho_{k}\right)$. That is, we use the centre of a circle and the radius to approximate the location and the size of sub-domains, respectively.

Example 2. The sub-domain with constant coefficient is a peanut or a peach or a pear or a bean. The exact centroid is $(0.1,0.3)$. Polar radius of a peanut is parameterized by

$$
r(t)=\frac{8}{25} \sqrt{(\cos t)^{2}+\left(\frac{\sin t}{2}\right)^{2}}, 0 \leq t \leq 2 \pi,
$$


polar radius of a peach is parameterized by

$$
r(t)=\frac{6}{25}-\frac{1}{15} \sin t-\frac{1}{35} \sin (3 t), 0 \leq t \leq 2 \pi,
$$

polar radius of a pear parameterized by

$$
r(t)=\frac{6}{25}+\frac{1}{20} \cos (3 t), 0 \leq t \leq 2 \pi
$$

and polar radius of a bean is parameterized by

$$
r(t)=\left(\frac{1}{5}+\frac{9}{50} \cos t+\frac{3}{100} \sin (2 t)\right) /\left(1+\frac{7}{10} \cos t\right), 0 \leq t \leq 2 \pi .
$$

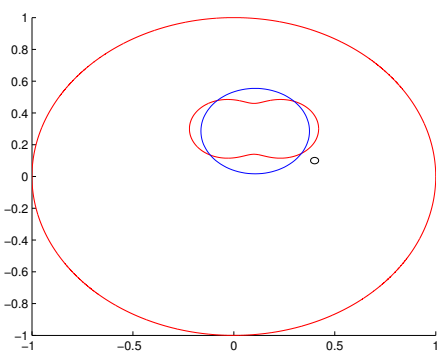

(a) $\alpha=0.2168, \varepsilon=10^{-5}$

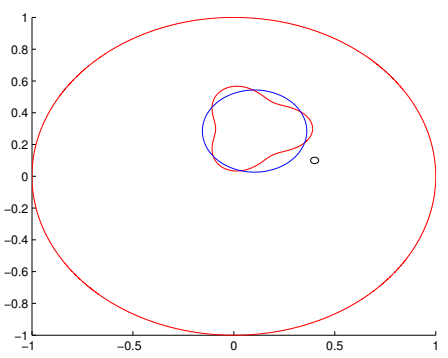

(c) $\alpha=0.2381, \varepsilon=10^{-5}$

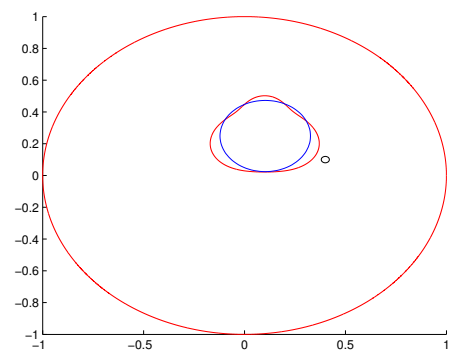

(b) $\alpha=0.4566, \varepsilon=10^{-5}$

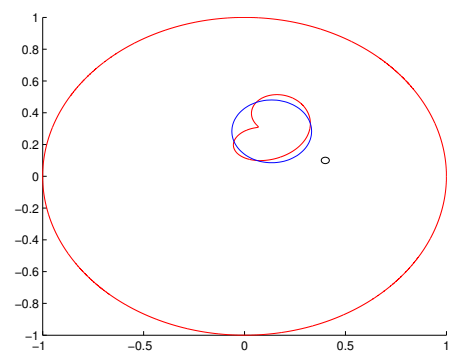

(d) $\alpha=0.3556, \varepsilon=10^{-5}$

Figure 3. (a) Peanut; (b) peach; (c) pear; (d) bean. Apply LMA to determine the location $O$ and the size $\rho$ of the coefficient along with exact solution (red), initial guess (black) and recovered solution (blue), respectively for Example 2

We apply LMA and TRA to determine the approximate centroid location and the size of the coefficient along with 0.01 noise data, see Figures 3 and 4 , respectively. To our proposed reconstruction algorithms, the starting guess is flexible and elegant. We take $(0.4,0.1,0.01)$ as a starting guess in Figure 3 and 4. From Figures 3, 4 and Table 2, we can see that the more accurate approximation of the location and the size is obtained for our different cases by LMA and TRA. 
Table 2. The approximate location and the size of the coefficient with piecewise constant using LMA and TRA with exact solution $(E S)$ and the starting guess $(S G)$ along with $1 \%$ noise data for Example 2

\begin{tabular}{lll}
\hline & (a) & (b) \\
\hline$E S$ & $(0.1,0.3, \rho)$ & $(0.1,0.3, \rho)$ \\
$S G$ & $(0.4,0.1,0.01)$ & $(0.4,0.1,0.01)$ \\
$L M A$ & $(0.1057,0.2857,0.2689)$ & $(0.1026,0.2484,0.2241)$ \\
$T R A$ & $(0.1050,0.2855,0.2686)$ & $(0.1024,0.2483,0.2241)$ \\
\hline & $(\mathrm{c})$ & $(\mathrm{d})$ \\
\hline$E S$ & $(0.1,0.3, \rho)$ & $(0.1,0.3, \rho)$ \\
$S G$ & $(0.4,0.1,0.01)$ & $(0.4,0.1,0.01)$ \\
$L M A$ & $(0.1026,0.2846,0.2586)$ & $(0.1346,0.2829,0.1975)$ \\
$T R A$ & $(0.1022,0.2844,0.2586)$ & $(0.1345,0.2828,0.1973)$ \\
\hline & &
\end{tabular}

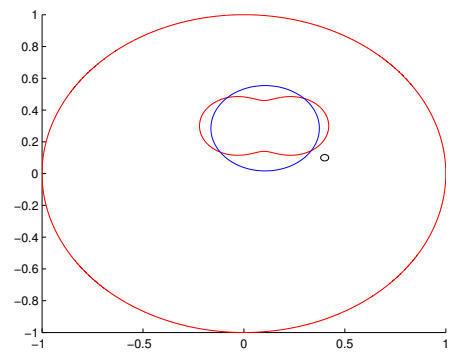

(a)

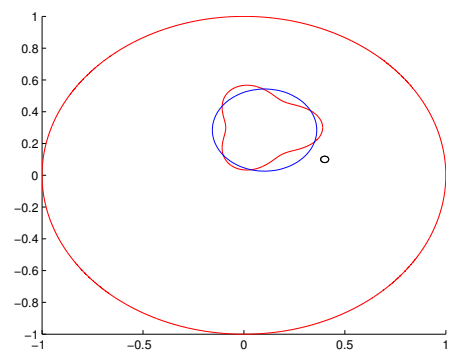

(c)

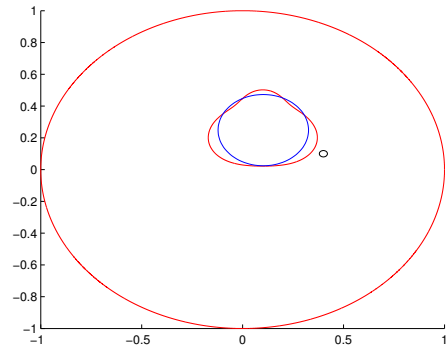

(b)

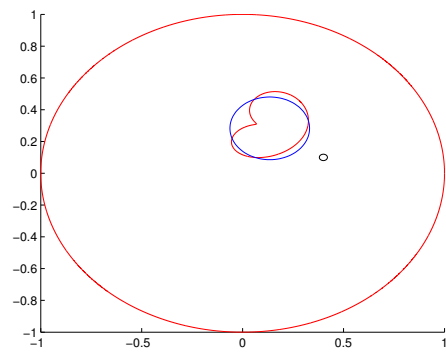

(d)

Figure 4. (a) Peanut; (b) peach; (c) pear; (d) bean. Apply TRA to determine the location $O$ and the size $\rho$ of the coefficient along with exact solution (red), initial guess (black) and recovered solution (blue), respectively for Example 2

Example 3. We consider two sub-domains with piecewise constant coefficient which are a peanut and a pear, and take the centroid as $O_{1}(-0.2,0.4)$ and 
$\mathrm{O}_{2}(0.1,-0.4)$. Polar radius of a peanut is parameterized by

$$
r(t)=\frac{4}{25} \sqrt{(\cos t)^{2}+\left(\frac{\sin t}{2}\right)^{2}}, 0 \leq t \leq 2 \pi
$$

and polar radius of a pear is parameterized by

$$
r(t)=\frac{3}{25}+\frac{1}{40} \cos (3 t), 0 \leq t \leq 2 \pi .
$$

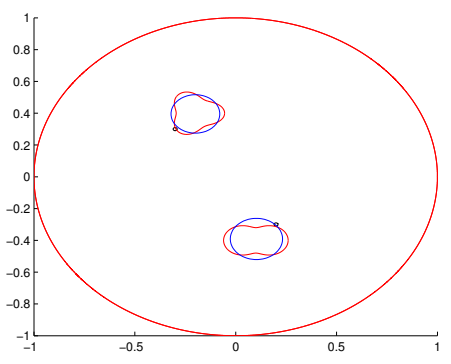

(a) LMA

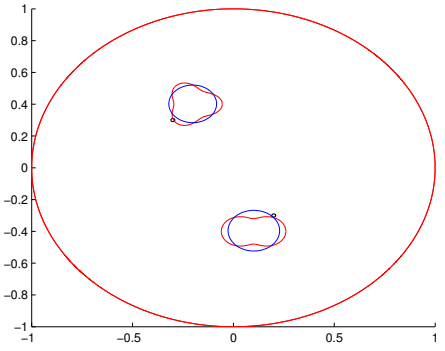

(b) TRA

Figure 5. Apply LMA and TRA to determine the location and the size of the coefficient along with exact solution (red), initial guess (black) and recovered solution (blue), respectively for Example 3

Table 3. The approximate location and the size of sub-domains with the constant using LMA and TRA with exact solution $(E S)$ and the starting guess $(S G)$ along with $1 \%$ noise data for Example 3

$\begin{array}{lll}E S & (-0.2,0.4, \rho) & (0.1,-0.4, \rho) \\ S G & (-0.3,0.3,0.01) & (0.2,-0.3,0.01) \\ (a) & (-0.2016,0.4021,0.1184) & (0.1014,-0.3956,0.1279) \\ \text { (b) } & (-0.1997,0.3955,0.1208) & (0.1028,-0.3912,0.1295)\end{array}$

We apply LMA and TRA to determine the location and the size with the same starting guess. In Figure 5, we can get the more accurate approximate centroid location and the size of sub-domains along with 0.01 noise data. From Figure 5 and Table 3, we know that we can obtain the better results for the location and the size of the coefficient with piecewise constant.

\subsection{Reconstructing the shape of the coefficient with piecewise con- stant}

In this sub-section, we apply LMA and TRA to recover the shape of the coefficient with piecewise constant. We assume that the number, the location and 
the size of sub-domains are known. The boundary $\Gamma_{k}$ of $\Omega_{k}$ is described as $O_{k}+r_{k}(t)(\cos t, \sin t)$, where $O_{k}$ is the centroid of the domain $\Omega_{k}$ and $r_{k}(t)$ is a real-valued function of $0 \leq t \leq 2 \pi$ along with $\beta_{k}=\left(c_{k, 0}, c_{k, 1}, \cdots, c_{k, 2 l_{k}}\right)$. We use $\beta_{k}$ to describe the shape of the piecewise constant coefficient for an inverse coefficient problem.

Example 4. We consider the shape of the coefficient is a peanut or a peach or a pear or a bean.

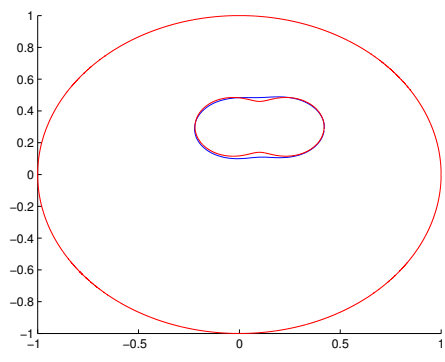

(a) $l=2, c_{0}^{(0)}=0.2686, \varepsilon=10^{-5}$

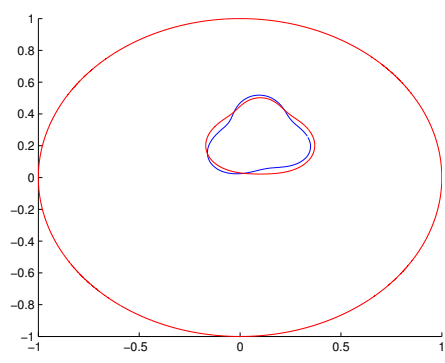

(b) $l=3, c_{0}^{(0)}=0.2241, \varepsilon=10^{-5}$

Figure 6. (a) Peanut; (b) peach. Apply TRA to reconstruct the shape of the coefficient with the accurate data along with exact solution (red) and recovered solution (blue), respectively for Example 4

In Figure 6, we take the location and the size as a starting guess in Table 2. Then we employ TRA to reconstruct the shape of the peanut and the peach along with the accurate data for Figure 6(a) and Figure 6(b), respectively. From Figure 6, we can see that we can not accurately reconstruct the boundary of sub-domain with constant coefficient even if we adopt the accurate data. However, our results are reasonable. We know that we use the computed solution to approximate the exact one, the calculation error is produced. Moreover, we discrete the system of integral equations, the discretization error also can produce. Thus the data in the process of iterations have some errors even if the starting input data are accurate.

In Figures 7 and 8, we take the location and the size as a starting guess in Table 2, then we employ LMA and TRA to recover the shape of the coefficient. For LMA, we take regularization parameters $\alpha=0.3124,0.7773,0.0290,0.0279$ for Figure 7(a), 7(b), 7(c) and 7(d) along with $\varepsilon=10^{-5}$, respectively. Figures 7 and 8 show that numerical algorithms work well to reconstruct the shape of the coefficient.

Example 5. We employ TRA to reconstruct the shape of two sub-domains with constant coefficient.

We try to reconstruct two sub-domains with constant coefficient using TRA. We take the location and the size from Table 3(a) as a starting guess to recover the shape of two sub-domains with constant coefficient in Figure 9(a). For Figure $9(\mathrm{~b})$, we fix the exact location at $(-0.1,-0.3)$ and $(0.5,0.1)$, take 


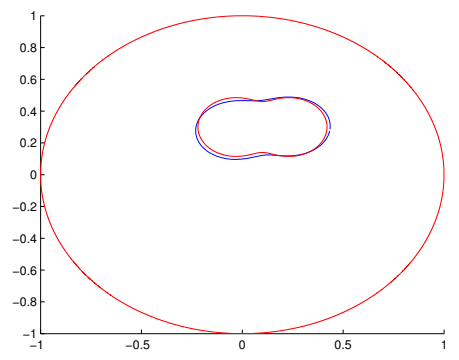

(a) $l=2, c_{0}^{(0)}=0.2689$

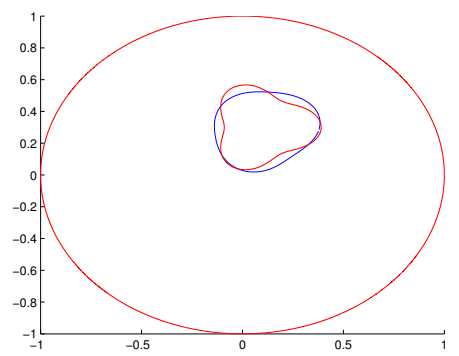

(c) $l=3, c_{0}^{(0)}=0.2586$

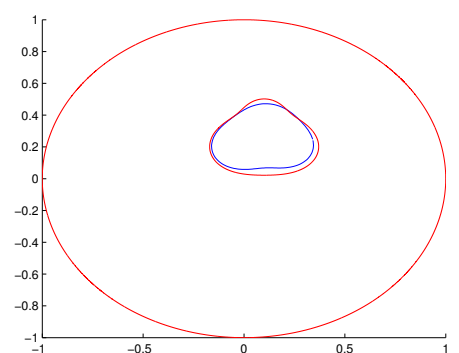

(b) $l=3, c_{0}^{(0)}=0.2241$

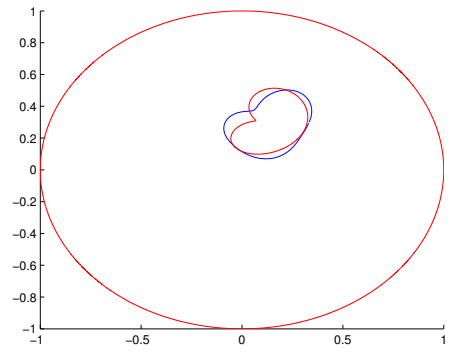

(d) $l=2, c_{0}^{(0)}=0.1975$

Figure 7. (a) Peanut; (b) peach; (c) pear; (d) bean. Apply LMA to reconstruct the shape of the coefficient with $1 \%$ noise data along with exact solution (red) and recovered solution (blue), respectively for Example 4

the size 0.01 as the starting guess, and then use TRA to reconstruct the shape of two sub-domains with constant coefficient. Figure 9 shows that our proposed numerical algorithm is effective to recover the coefficient with piecewise constant.

\section{Conclusions}

In this paper, we consider an inverse coefficient identification problem for the Schrödinger equation. We want to seek stable reconstruction algorithms to determine the number, the location, the size and the shape of the coefficient with piecewise constant form noise data on the boundary. Our advantage is to recover the shape of the coefficient with piecewise constant within a body along with the number unknown. This inverse problem is nonlinear and ill-posed. Thus we should consider regularization techniques in our proposed numerical algorithms. We propose two iterative regularization algorithms to detect the salient features of the coefficient with piecewise constant. Numerical examples show that the proposed reconstruction algorithms are efficient, feasible and stable to reconstruct sub-domains with constant coefficients. 


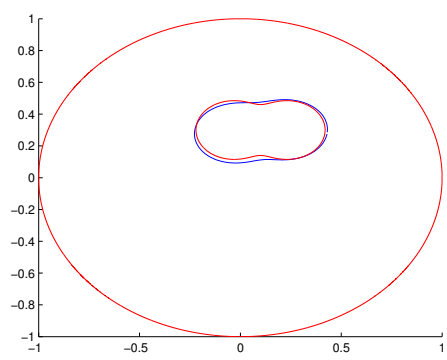

(a) $l=2, c_{0}^{(0)}=0.2686$

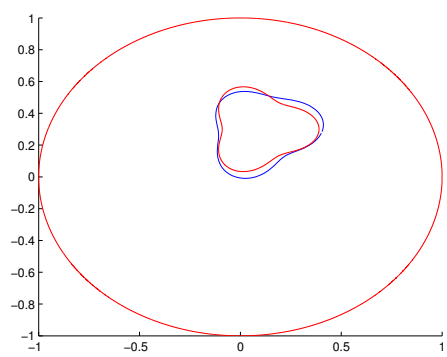

(c) $l=3, c_{0}^{(0)}=0.2586$

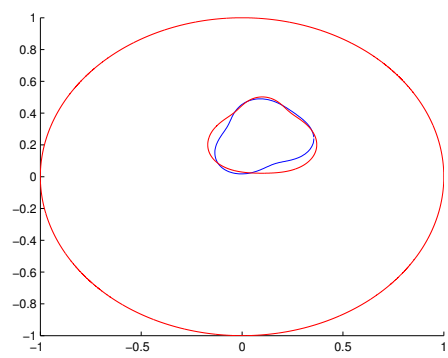

(b) $l=3, c_{0}^{(0)}=0.2241$

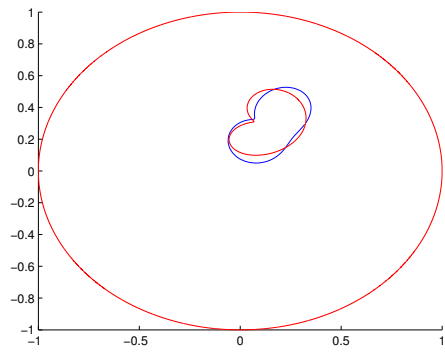

(d) $l=2, c_{0}^{(0)}=0.1973$

Figure 8. (a) Peanut; (b) peach; (c) pear; (d) bean. Apply TRA to reconstruct the shape of the coefficient with $1 \%$ noise data along with exact solution (red) and recovered solution (blue), respectively for Example 4

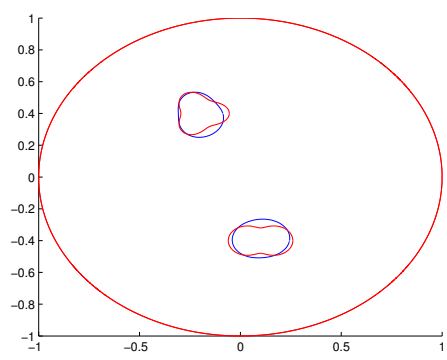

(a) $l_{1}=3, l_{2}=2$

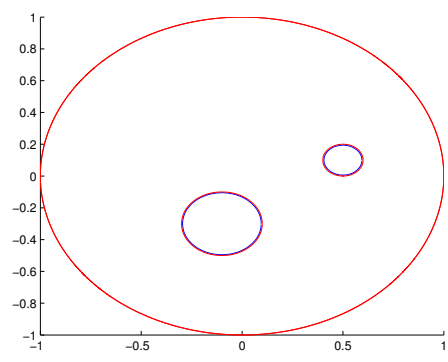

(b) $l_{1}=l_{2}=0$

Figure 9. Apply TRA to reconstruct the shape of the coefficient with piecewise constant along with $1 \%$ noise data along with exact solution (red) and recovered solution (blue), respectively for Example 5

\section{References}

[1] A. Altundag and R. Kress. On a two-dimensional inverse scattering problem for a dielectric. Applicable Analysis, 91(4):757-771, 2012. 
https://doi.org/10.1080/00036811.2011.619981.

[2] H. Ammari, E. Bonnetier, Y. Capdeboscq, M. Tanter and M. Fink. Electrical impedance tomography by elastic deformation. SIAM Journal on Applied Mathematics, 68(6):1557-1573, 2008. https://doi.org/10.1137/070686408.

[3] K. Astala and L. Päivärinta. Calderón's inverse conductivity problem in the plane. Annals of Mathematics, 126(1):265-299, 2006. https://doi.org/10.4007/annals.2006.163.265.

[4] X. Bai and B. He. Estimation of number of independent brain electric sources from the scalp EEGs. IEEE transactions on bio-medical engineering, 53(10):1883-1892, 2006. https://doi.org/10.1109/TBME.2006.876620.

[5] A.L. Bukhgeim. Recovering a potential from Cauchy data in the twodimensional case. Journal of Inverse and Ill-posed Problems, 16(1):19-33, 2008. https://doi.org/10.1515/jiip.2008.002.

[6] M. Cheney, D. Isaacson and J.C. Newell. Electrical impedance tomography. Industrial Tomography, 41(1):23-59, 2002.

[7] T.F. Coleman and Y. Li. On the convergence of interior-reflective Newton methods for nonlinear minimization subject to bounds. Mathematical programming, 67(1):189-224, 1994. https://doi.org/10.1007/BF01582221.

[8] T.F. Coleman and Y. Li. An interior, trust region approach for nonlinear minimization subject to bounds. SIAM Journal on Optimization, 6(2):418-445, 1996. https://doi.org/10.1137/0806023.

[9] R. Duraiswami, G.L. Chahine and K. Sarkar. Boundary element techniques for efficient 2-D and 3-D electrical impedance tomography. Chemical engineering science, 52(13):2185-2196, 1997. https://doi.org/10.1016/S0009-2509(97)000444.

[10] H. Eckel and R. Kress. Nonlinear integral equations for the inverse electrical impedance problem. Inverse Problems, 23(2):475, 2007. https://doi.org/10.1088/0266-5611/23/2/002.

[11] W. Fang and E. Cumberbatch. Inverse problems for metal oxide semiconductor field-effect transistor contact resistivity. SIAM Journal on Applied Mathematics, 52(3):699-709, 1992. https://doi.org/10.1137/0152039.

[12] B. Gebauer and N. Hyvönen. Factorization method and irregular inclusions in electrical impedance tomography. Inverse Problems, 23(5):2159, 2007. https://doi.org/10.1088/0266-5611/23/5/020.

[13] H.G. Gene, M.T. Heath and G. Wahba. Generalized Cross-Validation as a Method for Choosing a Good Ridge Parameter. Technometrics, 21(2):215-223, 1979. https://doi.org/10.1080/00401706.1979.10489751.

[14] B. Haberman and D. Tataru. Uniqueness in Calderón's problem with Lipschitz conductivities. Duke Mathematical Journal, 162(3):497-516, 2013. https://doi.org/10.1215/00127094-2019591.

[15] P.C. Hansen. Rank-deficient and Discrete Ill-posed Problems: Numerical Aspects of Linear Inversion. Society for Industrial and Applied Mathematics, Philadelphia, PA, USA, 1998. ISBN 0-89871-403-6. https://doi.org/10.1137/1.9780898719697.

[16] O.Y. Imanuvilov and M. Yamamoto. Inverse boundary value problem for Schrödinger equation in two dimensions. SIAM Journal on Mathematical Analysis, 44(3):1333-1339, 2012. https://doi.org/10.1137/11083736X. 
[17] O.Y. Imanuvilov and M. Yamamoto. Inverse boundary value problem for the Schrödinger equation in a cylindrical domain by partial boundary data. Inverse Problems, 29(4):045002, 2013. https://doi.org/10.1088/0266$5611 / 29 / 4 / 045002$.

[18] H. Kang. A uniqueness theorem for an inverse boundary value problem in two dimensions. Journal of mathematical analysis and applications, 270(1):291-302, 2002. https://doi.org/10.1016/S0022-247X(02)00085-9.

[19] R. Kohn and M. Vogelius. Determining conductivity by boundary measurements. Communications on Pure and Applied Mathematics, 38(3):289-298, 1984. https://doi.org/10.1002/cpa.3160370302.

[20] R. Kohn and M. Vogelius. Determining conductivity by boundary measurements II. Interior results. Communications on Pure and Applied Mathematics, 38(5):643-667, 1985.

[21] R. Kress. Linear integral equations, volume 82 of Applied Mathematical Sciences. Springer-Verlag, New York, second edition, 1999. https://doi.org/10.1007/9781-4612-0559-3.

[22] R. Kress and W. Rundell. Nonlinear integral equations and the iterative solution for an inverse boundary value problem. Inverse Problems, 21(4):1207, 2005. https://doi.org/10.1088/0266-5611/21/4/002.

[23] D.G. Luenberger. Introduction to linear and nonlinear programming, volume 28. Addison-Wesley Reading, MA, 1973.

[24] D.W. Marquardt. An algorithm for least-squares estimation of nonlinear parameters. Journal of the Society for Industrial \& Applied Mathematics, 11(2):431-441, 1963. https://doi.org/10.1137/0111030.

[25] V. Morozov. Methods of Solving Incorrectly Posed Problems. Springer-Verlag, New York, 1984. https://doi.org/10.1007/978-1-4612-5280-1.

[26] A. Nachman. Reconstructions from boundary measurements. Ann. of Math., 128(3):531-576, 1988. https://doi.org/10.2307/1971435.

[27] A.I. Nachman. Global uniqueness for a two-dimensional inverse boundary value problem. Annals of Mathematics, 143(1):71-96, 1996. https://doi.org/10.2307/2118653.

[28] G. Nakamura, Z. Sun and G. Uhlmann. Global identifiability for an inverse problem for the Schrödinger equation in a magnetic field. Mathematische Annalen, 303(1):377-388, 1995. https://doi.org/10.1007/BF01460996.

[29] H.H. Qin and F. Cakoni. Nonlinear integral equations for shape reconstruction in the inverse interior scattering problem. Inverse Problems, 27(3):563-648, 2011. https://doi.org/10.1088/0266-5611/27/3/035005.

[30] A.G. Ramm. A uniqueness theorem for a boundary inverse problem. Inverse Problems, 4(1):L1, 1988. https://doi.org/10.1088/0266-5611/4/1/001.

[31] J. Sylvester and G. Uhlmann. A global uniqueness theorem for an inverse boundary value problem. Annals of mathematics, 125(1):153-169, 1987. https://doi.org/10.2307/1971291.

[32] J. Sylvester and G. Uhlmann. Inverse boundary value problems at the boundarycontinuous dependence. Communications on pure and applied mathematics, 41(2):197-219, 1988. https://doi.org/10.1002/cpa.3160410205. 